\title{
Demarcating cognition: the cognitive life sciences
}

\author{
Fred Keijzer ${ }^{1}$
}

Received: 24 July 2018 / Accepted: 14 July 2020 / Published online: 15 September 2020

(C) The Author(s) 2020

\begin{abstract}
This paper criticizes the role of intuition-based ascriptions of cognition that are closely related to the ascription of mind. This practice hinders the explication of a clear and stable target domain for the cognitive sciences. To move forward, the proposal is to cut the notion of cognition free from such ascriptions and the intuition-based judgments that drive them. Instead, cognition is reinterpreted and developed as a scientific concept that is tied to a material domain of research. In this reading, cognition becomes a changeable theoretical concept that can and must be adapted to the findings within this target domain. Taking humans as the best-established existing example of the relevant material target domain, this central case is extended to include all living systems. To clarify what it is about living systems that warrants their role as cognitive target domain, the new concept of cobolism is introduced as a complement to metabolism. Cobolism refers to the systematic ways in which each living system encompasses structures, processes and external events that maintain the fundamental metabolic processes that constitute the core of each living system. Cobolism is perfectly general, applies to bacterial and human cases alike, and provides a general format to describe wildly different cognitive organizations. It provides a clear target for the cognitive sciences to work on, turning them into what we can call the cognitive life sciences.
\end{abstract}

Keywords Cognition - Cognitive science $\cdot$ Mark of the cognitive $\cdot$ Cognitive domain $\cdot$ Biocognition $\cdot$ Cognitive biology $\cdot$ Cognitive life sciences

Fred Keijzer

f.a.keijzer@rug.nl

http://www.rug.nl/staff/f.a.keijzer/index

1 Faculty of Philosophy, University of Groningen, Oude Boteringestraat 52, 9712 GL Groningen, The Netherlands 


\section{Introduction}

The cognitive revolution and the subsequent rise of the cognitive sciences ${ }^{1}$ initiated a new scientific approach to the study of mind. This approach introduced concepts such as information processing, computation and representation, and applied them to produce a fully naturalized account of various mental processes that previously seemed beyond a clear natural science approach (e.g. Gardner 1987; Green 1996; Boden 2006). Within the cognitive sciences, the notion of 'cognition' became a standard term to refer to its target, which initially consisted only of topics like high-level human thought and language, but which widened over the years. 'Cognition' provided a scientific, naturalistic phrase that stressed a modern non-dualistic view on the mind that could be articulated in terms of information processing and computation. Nevertheless, the new scientific domain referred to as cognition remained intrinsically bound up with the pre-existing and long-standing notion of the mind.

For a long time, the empirical extension of cognition was not explicitly articulated but simply taken to coincide with that of the mental processes targeted. Intuitive, common-sense judgments would suffice to establish the presence of these processes, as for example in Turing's influential proposal for a way to test whether machines can think (Turing 1950). Turing's test is relevant here as an illustration of two widespread assumptions. First, the test accepts that the presence of a mental process can be established by an ordinary human, in this case by judging the plausibility of answers given to questions posed by this human. Second, the test dismisses the relevance of the general physical characteristics of the organization answering these questions. Thus, the presence of mental and cognitive processes can be established in a low-tech way by ordinary persons, while material constitution plays a small role.

In this context, the perceived need to specify clear and specific criteria-a mark-for the organizations or processes to which cognition applies was limited. Andy Clark shows the mindset:

There is no need for a mark of the cognitive [...] because we already have an implicit (though probably unformalizable in words) grip on the coarse-grained behavior patterns that we take to be indicative of key mental states (Clark 2011, p. 451)

The empirical presence of cognition is here taken to be intuitively given and signaled by a broad variety of implicitly plausible criteria, such as Clark's "coarse-grained behavior patterns".

Nowadays, such an intuitive demarcation of cognition based on the ascription of mind has become less self-evident as 'cognition' has come to refer to a much broader set of phenomena. These do not only include thought, memory and language, but also perception, emotion, motivation and motor control (Akagi 2018). In addition, developments within the cognitive sciences such as neural networks (Rumelhart and McClelland 1986), dynamical systems (Van Gelder 1995), embodied approaches (Hurley 1998), enactivism (Varela et al. 1991) and cognitive neuroscience (Poldrack

\footnotetext{
${ }^{1}$ I will throughout use the plural 'the cognitive sciences' to refer to the various and (sometimes very) different disciplines and sciences that nevertheless belong together because they share an overarching domain.
} 
2010; Anderson 2015) have stretched the usage of 'cognition' even wider. Given these changes, can we still rely on intuitive common-sense judgments to demarcate the cognitive domain?

Clark's - and many others' - easy reliance on an intuitive recognition of cognitive processes is currently challenged and discussed in various debates. An important early example centered on the extended mind idea (Clark and Chalmers 1998), which provoked a call to formulate "a mark of the cognitive" to clarify what cognition is (Adams and Aizawa 2001; Adams and Garrison 2013; Clark 2008, 2011; Rowlands 2010). The call to clarify cognition became subsequently a wider concern that received a lot of attention (e.g. Walter and Kästner 2012; Kaplan 2012; Serrano et al. 2014; Buckner 2015; Newen 2017; Ramsey 2017; Allen 2017; Akagi 2018; Figdor 2018; Bayne et al. 2019). Discussions on 'cognitive ontology' also made an impact on the cognitive neurosciences (Poldrack 2006, 2010; Figdor 2013; Anderson 2015) and by now the position in the quote reflects a specific position rather than the consensus view.

Central issues in the various debates are (a) the ongoing changes in the notion of cognition itself; (b) the very need and the possibility of a clear demarcation of cognition; and (c) formulating potential criteria-marks of the cognitive-for such a demarcation. So far, no clear consensus has emerged on any of these three issues, nor is such a consensus in sight. In this paper, I want to make progress on all three issues by asking a meta-question first: Why is it so hard to reach a consensus concerning the domain of cognitive science?

The answer developed here builds on two ideas that are recently brought forward within the cognition discussions. First, cognition, as used within the cognitive sciences, must be considered a changeable theoretical term that has to be explicitly developed as such (Akagi 2018). Within the cognitive sciences, the concept of cognition has been enriched and developed on the basis of empirical findings and theoretical developments. Akagi considers such conceptual progress the "hard-won fruits of scientific inquiry [... that] pushed against pretheoretic intuitions" (p. 3555). Counterintuitive claims concerning cognition are not a vice when we aim to understand it scientifically. In the following, cognition will be explicitly cast as such a theoretical term that can develop in ways that diverge from its initial meaning. This raises the question how these changes can be reconciled with existing intuitions concerning mind and cognition that limit new ways to conceptualize cognition.

Second, cognition, as used within the cognitive sciences, should refer to an empirical domain constituting the target of explanation (Ramsey 2017; Allen 2017). Ramsey argues that the demarcation of cognition should not be done by "appealing to explanatory strategies, methodologies, or theoretical commitments" (p. 4207). Instead, cognitive science "should adopt the standard way sciences are defined" that is "in terms of what it is that is being studied". Ramsey mentions geology and chemistry as examples where the empirical domain defines the sciences. Similarly, "Cognitive science should be defined by the target of the explanation" (p. 4208).

While taking in Ramsey's idea of the need to provide an explicit target domain for the cognitive sciences, I will diverge from his proposal what this empirical target domain (initially) might be, namely "our intuitive, pre-theoretical image of the mind" (p. 4208). Setting up the mind as demarcating "what it is that is being studied" by 
the cognitive sciences is insufficient. First and discussed in the next section, there is a growing set of empirical findings that arguably belong within the cognitive sciences without fitting an intuitive image of mental processes. Second, mental processes do not designate an empirical target domain that is independent of "theoretical commitments". In other sciences, such as geology and chemistry, the material domain is primary and empirical measurements and results concerning this material domain ultimately will drive theory building and the resolution of controversies. As the example of Turing's test illustrates, casting the cognitive domain empirically in terms of 'things that think' brings in deep theoretical commitments that dissociates this domain from its actual material constitution, as the long history of functionalism bears witness to. This line of thinking also prevents taking any specific material domain- even the brain-as a necessary condition for cognition, as long-standing views about mind and cognition oppose such a 'restrictive' interpretation. However, this focus on necessary, let alone sufficient conditions for the application of a concept is misguided when it comes to the empirical study of cognitive phenomena. To make sense of such phenomena, we can simply study the cases that are present in the world and work from there. The influential assumption that we already know what cognition is — generally speaking — and that we are therefore able to make sound, a priori scientific decisions concerning the empirical targets that are exhibiting it must be challenged. As will be discussed below, this assumption insulates the study of cognition from a material empirical basis from which we can learn and improve our understanding of these phenomena. Thus, using common-sense ascriptions of mind does not provide a satisfactory empirical domain for the cognitive sciences that fits in with Ramsey's proposal. Instead, we will take this domain to consist of specific material organizations.

By combining both ideas (cognition is a theoretical term and cognition is tied to a particular material domain) the outline of a reply to the meta-question becomes visible. In both cases, there is a tension between theoretical and empirically driven changes to either the notion of cognition or the domain it refers to on the one hand, and on the other an opposing tendency to remain within the confines of what cognition is traditionally about, most notably higher-level human thought, memory and language.

The proposal developed below sets aside intuitive common-sense demarcations of cognition which are closely linked to mind. By dissociating the demarcation of mind - still based on intuitive human judgments - and of cognition—now referring to processes within a suitable material target domain-it becomes possible to move forward on the three main issues that feature in current discussions on the demarcation of cognition and defend the following positions: (a) yes, the notion of cognition is indeed changing and therefore; (b) it becomes essential to develop a clear way to constrain our interpretation of what we take cognitive phenomena to be; and (c) this demarcation can be achieved by reinterpreting cognition as a theoretical term that is meant to reflect the features of a material target domain. For reasons discussed below, this domain will be cast as consisting of living systems, which encompasses humans, animals, and many atypical forms of cognition.

The text has the following structure. Section 2 introduces new empirical and theoretical targets for the cognitive sciences that challenge a human and mind-centered interpretation of cognition. Section 3 discusses intuitive mind-oriented demarcations of cognition and how they hinder a systematic material interpretation of cognition. 
In Sect. 4, the concepts of mind-as an intuition-based term-and cognition-as specifying the target domain for the cognitive sciences-are dissociated, allowing an independent scientific role for the notion of cognition. Sections 5 to 7 subsequently develop the proposal that living systems provide a suitable material target domain for the cognitive sciences.

\section{Atypical targets for the cognitive sciences}

Ongoing research in a broad variety of fields provides important empirical reasons to be critical about a demarcation of cognition based on common-sense judgments concerning mental states and processes. The findings concern cases that are arguably examples of cognition, while common-sense judgments tend to exclude them. The phenomena are relevant to the cognitive sciences because they involve features like sensing, valence, behavior, memory, learning, anticipation, decision making and communication, which in humans would suffice to ascribe cognition. Lyon (2015) regards these phenomena as elements of a cognitive toolkit that many, or even all, organisms have available. They provide a clear set of cognitive explananda.

The presence of such toolkit features will here be taken as a pragmatic way to signal cognitive relevance, if not cognition. Even Adams, who defends a conservative interpretation of cognition, acknowledges the presence of "a level of explanation that rises above the basic cellular and mechanical levels of explanation of biology" (2018, p. 29). Irrespective whether we tend to ascribe cognition or even mind, to these cases, the organisms involved exhibit features that normally signal the presence of cognitive processes, features that allows them to deal with their environments, keeping them alive and reproducing. Such toolkit features require dedicated scientific attention from within the cognitive sciences to understand them. I will refer to these non-standard cases as atypical forms of cognition. A short overview of the relevant research will illustrate how common-sense ways of demarcating the cognitive domain run into difficulties here.

The first thing to note is the cognitive status of all animals (e.g. Keijzer 2001; Carruthers 2004; Lyon 2006; Godfrey-Smith 2002; Chittka 2017). Animal behavior involves perceptual and motoric skills that constitute major cognitive achievement, something that becomes self-evident when one tries to mimic similar capacities in robotic devices (Pfeifer and Bongard 2007). As Godfrey-Smith says:

But when a fish negotiates its way around a reef, or a rat finds its way back to a food source, the internal processes responsible for these behaviors are varieties of cognition as well. (2002, p. 224).

While there are enormous differences in complexity and organization between bonobos and pill bugs, both have complex sensorimotor systems and an array of normative constraints to respect to stay alive and reproduce. Animals are not at heart passive and basic stimulus-response machines (Brembs 2009, Keijzer et al. 2013, O'Regan and Noë 2001) but complex cognitive beings that score high on the toolkit's criteria. 
Similar stories can be told for bacteria, protists, plants and fungi. Bacteria exhibit a wide array of cellular adaptations to environmental circumstances for tracking environmental features and enabling complex social interactions (Macnab and Koshland 1972; Ben-Jacob et al. 2004; Westerhoff et al. 2014; Lyon 2015). There is also an active research line targeting slime molds, belonging to the Protista, that can negotiate mazes (e.g. Reid and Latty 2016). Examples of cognitive toolkit phenomena in plants go back to Darwin and have now become a lively research domain (e.g. Baluška et al. 2009; Trewavas 2005; Mancuso and Viola 2015; Van Loon 2016). Plant growth involves decision-making behavior that takes large numbers of different and difficult to reconcile constraints into account. The fungi provide their own examples, such as the directional growth of their filaments, which is sensitive to environmental circumstances, while the extending tips send signals to the nucleus that controls subsequent development (Etxebeste and Espeso 2016). Complex signaling also takes place between fungi and plants for example when the fungus grows specialized organs inside appropriate plant cells (Parniske 2008).

Atypical cases are not restricted to a recognizable agent-environment context. There are also examples from within the body that I will refer to as inbodied cognition. This involves phenomena like bodily development, change and regeneration (Baluška and Levin 2016). Using a computational perspective on body growth, maintenance, and regeneration after damage, Mike Levin and his group have established features like a 'bioelectric code' that coordinates cell development and tissue patterning and provides goal states for body shape (Levin 2013; Levin and Martyniuk 2018). One example is the possibility to change a bioelectric membrane code of planarians that changes their body form. When such animals are cut in half, they now regenerate with either two heads, two tails or other new 'goal states' for the fully-grown body. When cut anew, they again grow two heads or tails and so on, until the manipulation is reversed and the 'normal' body form regrows. A different case consists of decision-making behavior by the cells constituting blood vessels. These cells act in a highly complex and dynamic tissue-environment where they start new blood vessels under the proper circumstances (Bentley et al. 2014; Bentley and Chakravartula 2017).

Generating, maintaining and adapting our animal body organization must be considered even more atypical than the previous atypical cases. Still, these processes exhibit toolkit features that are regular cognitive explananda. These cases are also open to computational approaches and fitting targets for the cognitive sciences (Manicka and Levin 2019; Bentley et al. 2014). At this point, it is also important to keep in mind that neurons - which are generally considered to be cognitively relevant-are not fundamentally different from other cells, while nervous systems do more than organize behavior (Jékely et al. 2015). All in all, there are good reasons to consider the possibility that inbodied cognition is a widespread phenomenon.

Atypical cases of cognition provide a major and new challenge for the cognitive sciences that needs to be addressed. The question is how. Most of these cases are intuitively outside the domain normally addressed with words like mind and cognition but there is hardly a suitable alternative vocabulary. As Davidson once phrased the issue of boundary cases: 
We have many vocabularies for describing nature when we regard it as mindless, and we have a mentalistic vocabulary for describing thought and intentional action: what we lack is a way of describing what is in between. This is particularly evident when we speak of the 'intentions' and 'desires' of simple animals; we have no better way to explain what they do" (Davidson 1999, p. 11).

We can no longer accept this lack of options for describing natural "in between" phenomena that exhibit a broad spectrum of cognitive toolkit criteria. Irrespective whether we intuitively ascribe mind or cognition to these cases, they provide interesting and even important targets for the cognitive sciences. Atypical cognitive phenomena make it an urgent matter to expand our views on what we take cognition to be.

\section{3 "But is it really cognition?"}

Should these atypical cases-signaled as cognitive explananda by the presence of cognitive toolkit features-be accepted as real cognitive phenomena? For many philosophers and scientists, it will be absolutely clear and even absurd to say that atypical cases like plants, bacteria or blood-vessel cells have a mind or exhibit cognition. On the other hand, the scientists working on these atypical cases and some of the philosophers reflecting on them come to a different conclusion and do argue for their inclusion in the cognitive domain.

The best way to end scientific controversies is by providing clear empirical evidence that settles the dispute. To mention a classic example, continental drift initially seemed preposterous and counterintuitive to many scientists as it seemed inconceivable that whole continents could move across the globe. However, as the concept 'continent' clearly referred to major landmasses on Earth and empirical evidence concerning these landmasses accumulated, existing preconceptions had to be set aside. Nowadays, the concept 'continent' stands for landmasses that travel the Earth, while breaking up and colliding again like flotsam. Of course, many cases are less straightforward, but collecting empirical evidence and using it to decide on diverging judgments is the key to reaching consensus in science.

The cognitive sciences have a problem when it comes to providing unequivocal material evidence bearing on their target. Returning to the example of Turing's test, the presence of mental processes and cognition can be decided by ordinary human observers, and is not due to the specific material constitution of a system. For the cognitive neurosciences, the material system plays a larger role, but even here the perceived role of the physical brain derives from its role as a realizer of mental processes and cognition. The presence of mental or cognitive processes in any particular system is not seen as necessarily tied to and dependent on a specific material constitution that can enforce major changes in how we interpret cognition.

Deciding whether an organization, an organism or artifact, exhibits mental or cognitive features is based on intuitive judgments that are influenced by at least two, often opposing, tendencies. On the one hand, there are fast and automatic tendencies to ascribe mental and cognitive processes to almost anything that moves or talks. Humans ascribe feelings to a robotic device being kicked (e.g. Barrett 2015), while 
Fig. 1 Ascribing mind or cognition to specific (im)material organizations is prone to opposing tendencies to include or exclude potential cases as actually exhibiting mind or cognition

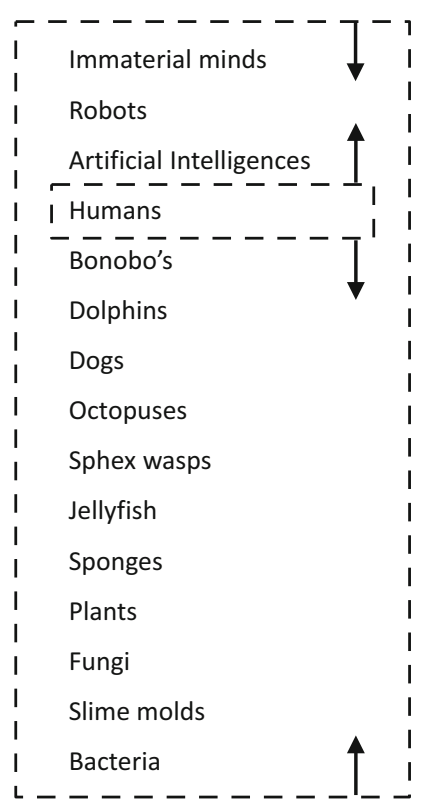

animacy-research shows how we ascribe mental states and actions to targets that show simple self-initiated movements (e.g. Scholl and Tremoulet 2000; Gao et al. 2009). This tendency to ascribe mind and cognition widely is countered by deliberate philosophical theorizing that aims to articulate, organize and evaluate such intuitive judgments. Unfortunately, the results of these deliberations do not converge. For example, Burge names representational states as the relevant condition for mind, which would include insects (2014, p. 400). More restrictive proposals take the use of concepts as a requirement, suggesting that non-human animals in general do not even perceive or act (Hamlyn 1994; Stoecker 2009). Others criticize the idea that concepts are a requirement for mind (e.g. Carruthers 2004), or claim that some non-human animals do use concepts (e.g. Hurley and Nudds 2006). No consensus has been reached when it comes to deciding which organizations really have a mind or really exhibit cognition.

Figure 1 shows a range of systems that can be considered to exhibit mind or cognition to some extent. The immaterial minds are included to signal that being physical is not a necessary condition for these concepts to apply. Only humans are universally accepted, while in all other cases it is a matter of debate whether they really exhibit mind or cognition. For example, depending on the interpretation, bonobos and New Caledonian crows may be included as systems exhibiting cognition, but it may also be that additional, more stringent considerations will exclude them again. For this reason, these animals do not provide an unequivocal, materially designated target for the cognitive sciences that is independent from theoretical commitments. Rather, mental (and cognitive) processes are the target, while these animal cases count only if and, if so, as far as they exemplify or realize such processes. The result is a concept-driven instability concerning the empirical target domain for the cognitive sciences. 
Under these circumstances, our understanding of mind and cognition remains cut off from a wide range of empirical feedback because potential counterexamples of certain views can for this very reason be excluded from the domain of interest. To see this, it is useful to think in terms of two directions of fit (Searle 1983). Given the presence of two sides that are supposed to match - the concept of cognition and the organizations in the world that exhibit cognition-either of the two can be dominant while the other follows. Given a concept-to-world direction of fit the notion of cognition comes first and the organization in the world must fit the concept to be accepted as really exhibiting cognition. In the reverse world-to-concept case, the empirical organization is dominant and the concept must be adapted to accommodate the features of the investigated organization.

To illustrate the difference, consider bacteria that use a molecular memory to orient themselves. Working with a concept-to-world fit, the question is whether such bacteria 'really' exhibit something that we can acknowledge as a memory, or merely something that looks like it. In the latter case, the bacterial example could be considered as falling outside the domain of interest and be excluded from consideration. With a world-toconcept fit, the material bacterial case is primary and the bacterial use of something that acts as a memory would require us to accommodate our views as to what memory might be. Ramsey's examples of geology and chemistry mentioned above also exhibit such a world-to-concept direction of fit. In practice, both directions of fit are important and interact in doing science. We need concepts and theories to guide empirical work, while the latter provides a testing ground for these concepts and theories, often leading to conceptual changes. However, in specific cases their relative influence can differ.

For the cognitive sciences, concept-to-world thinking is very influential. The intuitive ascription of mind and cognition gives precedence to cognition-related concepts rather than the entities that potentially exhibit them. In addition, the lack of consensus as to where the concepts apply makes for a very unstable cognitive domain that provides only limited guidance for updating and adapting cognition-related concepts to accommodate specific empirical findings. Atypical cases of cognition that could potentially change our views on cognition can be kept out of consideration without a clear material target domain. In this respect the cognitive sciences remain themselves atypical, compared to other sciences.

\section{Cutting the conceptual link between mind and cognition}

This is the analysis so far: Within the cognitive sciences, articulating the domain of cognition is heavily debated, so far without clear results. New findings concerning atypical cognitive phenomena also need to be dealt with. A main problem here is that long-standing interpretations of mind and cognition become less useful from a cognitive science perspective, while they remain difficult to change as they remain relatively insulated from empirical critique. Given the analysis in terms of direction of fit, a natural solution is to reverse cognitive science's dominant concept-to-world fit. The conceptualization of mind and cognition could be made to reflect empirical and related theoretical considerations much more extensively and rigorously than before. 
Fig. 2 Human cognition is usually cast in the context of mental phenomena, which would include cognition. Here, mind and cognition are dissociated even when humans fit both. In this way, not all cognitive phenomena need to fit the criteria for ascribing mind

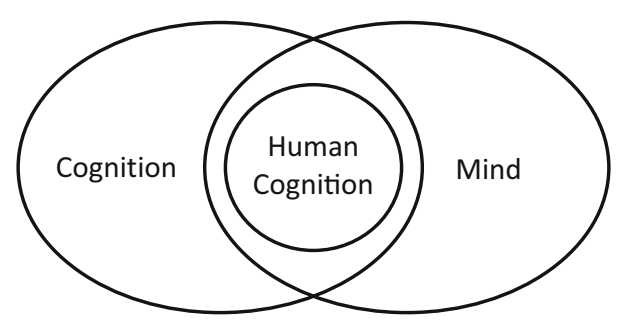

Thus, can we turn mind and cognition into empirical scientific concepts that will be adapted to scientific findings and theorizing?

For mind, I would say no. Mind is a key concept within our culture that is central for many topics ranging from responsibility, free will, using reasons, being rational, and so on. This usage extends far beyond current scientific concerns. We should not consider changing its present usage in any radical way. For the concept of cognition, the situation is different. Cognition has already developed into a modifiable theoretical term within the cognitive sciences, as well as a conceptual marker of the cognitive domain. The existing difficulties in accepting changes to what we take cognition to be (leave alone adding more changes) derive from an intuitive demarcation that still remains close to that of mind. Major changes in our views on cognition are associated with major shifts in how we interpret our own minds.

At this point, I propose a straightforward though radical way to proceed: cut the conceptual link between mind and cognition and start using them as two independent concepts that, over time, can come to reflect very different meanings (Keijzer 2017). In this proposal, mind keeps its current meaning and usage, and will be left out of further consideration here. In contrast, cognition will be explicitly interpreted as a theoretical scientific concept that can —or rather must—be adapted to the material domain that constitutes its scientific target.

Cutting the conceptual connection between mind and cognition sets our interpretation of cognition free from an intuition-based conceptualization and turn it into an empirically-driven concept. The idea is visualized in Fig. 2, where human cognition is taken as the central case, but now set in two different domains. On the cognition side, human cognition is positioned among other systems exhibiting cognition, irrespective whether these have a mind or not. On the mind side, human cognition is interpreted as an aspect of mind and positioned within a different range of organizations, not all of them natural (see also Fig. 1).

Separating cognition from its intuition-based conceptualization does not imply that a new interpretation of cognition needs to be built on the spot or from scratch. Nor need the cognitive sciences to be changed overnight. Obviously, high-level cognitive tasks as exhibited by humans will keep their place as a central concern. However, by using a bootstrapping process that accommodates a wider variety of findings - such as those from atypical cases - the human case can be gradually fitted into this wider context. As an analogy, one can think of two boats previously tied together that are cut loose. The two boats will initially be in the same area but from here on become capable of traveling into different directions, moving apart gradually. 
Tying the cognitive sciences to a specific material domain involves a fundamental change in the way we approach the study of cognition. What used to be mere 'realizers' now become the empirical starting point. Relatedly, instead of assuming a priori that the main characteristics of this cognitive domain are known, this becomes an issue that is open to investigation. As Allen phrased it spot on: "enquiry should not be stifled by a conservatism about terms and their meanings that insists on stipulating what we are studying before we study it" (2017, p. 4241). Cognition requires further clarification by empirical and theoretical study.

The present proposal relates to and extends recent ideas from Akagi (2018), and from Ramsey (2017) and Allen (2017). Like Akagi, it takes cognition to be a theoretical term that can be changed and adapted to the scientific needs at hand. What it adds here is the dissociation between mind and cognition, which allows more freedom in changing what we take cognition to be. In line with Ramsey and Allen, the present proposal also stresses the need to focus on empirical cases to develop our understanding of cognition. Of these two, Allen is critical about defining cognition, considering the term too general in comparison to more specific cognitive capacities, while such definitions are often used to limit the cognitive domain. I agree. The present proposal does not define cognition but sets it up to reflect a material domain, which fits Allen's view that we should focus on empirical results. In contrast to Allen's view, I maintain cognition as a general and integrative label to help tie together an increasingly divergent agglomeration of cognitive sciences that will increasingly target atypical cases.

Concluding, the proposal presented here separates cognitive phenomena from their current intuition-based demarcation and association with the mind. Instead, cognition is taken to refer to a material domain that provides the proper target for the cognitive sciences, and which can diverge sharply from the mental domain. There is now one crucial question left: What might this material domain be that the cognitive sciences are to be the study of?

\section{Specifying a suitable target domain: zooming in}

Specifying a plausible material target domain for the cognitive sciences does not require a sophisticated theoretical specification of cognition. The target domain itself should constrain and guide such a specification, as it did for our understanding of continents and many other topics. A standard scientific bootstrapping process where theorizing and empirical work coevolve will be a much surer way to progress than conceptual stipulation. Still, to get started, a target domain must be chosen, which involves some initial theoretical decisions (that always remain open to reevaluation). Here, I discuss several points of departure that together provide the outline for a suitable target domain.

A first point of departure derives directly from the dissociation between mind and cognition as proposed above: We have the theoretical freedom to question and set aside long-standing intuitive ideas concerning cognition. These ideas will still influence us explicitly and implicitly, but when attended to, they need not guide our judgment or limit changes in how we interpret and think about cognition. 
Second, the cognitive domain should include the widely diverging atypical cases that provide relevant targets for (some of) the cognitive sciences. Atypical cases are also not easy to recognize 'on sight' and have a long history of neglect. Given their late recognition, it will be essential not to exclude potential cases beforehand. Atypical cases of cognition act as a gamechanger for the cognitive sciences as we will need to include these cases in the cognitive domain to decide on their status in an informed way.

Third, a materially designated domain must include humans. However, including humans as material organizations is not limited to high-level cognitive features as Turing's test highlighted, or even just the human brain. Instead it involves complete human bodies, which are highly complex living systems. The latter immediately opens up a conceptual and empirical highway that connects the human case with living systems more generally of which humans are just one example. Humans are complex bilaterians - the largest animal grouping — and their structural, developmental and physiological characteristics reflect this evolutionary ancestry (Rosslenbroich 2014). Animals, in turn, are multicellular organizations, some of them quite large, which are constituted by a variety of cell types and extracellular materials. At a deeper cellular and biomolecular level of organization, humans are increasingly similar to other living systems and when it comes to foundational features-such as DNA and the ATP synthase-we are constructed from the same ingredients and depend on the same fundamental processes as all known lifeforms (e.g. Lane 2016). Given the general principle of biological continuity, extending the cognitive domain to include all living systems is a plausible extension that also provides a methodologically workable domain that is inclusive and easy to demarcate without major boundary issues.

Claims to extend cognition to living systems generally have been made before, such as Maturana's "Living systems are cognitive systems," (Maturana and Varela 1980, p. 13, emphasis in original), Stewart's (1996) "cognition = life" formulation, and Lyon's (2006) biogenic approach, which states that the study of cognition should begin with basic biological principles and is best seen as a biological function (Lyon 2019). At the same time, such claims have not been widely accepted, as witnessed by the current state of the debate on cognition (Sect. 1). The switch to a world-toconcept direction of fit provides a conceptual instrument to counter influential intuitive judgments that separate cognitive phenomena conceptually from living systems even when their empirical connection is pervasive.

As a fourth point of departure, taking living systems as the material target domain, one might consider drawing a boundary at the skin or cell surface. However, this is a major limitation that must be avoided. Living systems do not end at such boundaries as they require a medium to live and act in (e.g. Thompson 2007; Di Paolo et al. 2017). Lyon's toolkit requirements also stress issues relating to interaction with an environment, while external features are central to human cognition with their use of artifacts and their social and cultural practices. Given the importance of embodiment, sensorimotor skills, and extensive forms of niche construction, the cognitive domain cannot be limited to (collections of) cells and their products.

A final point of departure states that cases of artificial intelligence or AI are problematical. AI cases are tailored to fit human intuition-based judgments concerning cognition, a criterion set aside here. In addition, the material organization and way 
of operation of AI cases is very different from humans and living systems generally. Adding them to the target domain implies that we know enough about cognition to dismiss these differences, which is precisely what is challenged here. For example, if AI cases are accepted, a heat-seeking missile can be used as a counterexample to, for example, a theoretical account based on the idea that metabolic processes are central to cognitive phenomena (Degenaar and O'Regan 2017). Given the prominence of AI cases in the current cognitive sciences, must we include them in the cognitive domain?

A reason to exclude AI cases from the domain can be drawn from Godfrey-Smith's (2016a) useful differentiation between coarse-grained and fine-grained organizational features. In this case, AI's current coarse-grained focus on global system behavior like perception and decision-making does not incorporate the fine-grained biomolecular profile of living systems and arguably cannot incorporate such a sufficiently finegrained profile:

In the living system, the information-processing side of its activity is integrated with the metabolic side, so the two can only share coarse-grained functional properties. (Godfrey-Smith 2016a, p. 502, emphasis added)

As long as we are not sure how a coarse-grained focus influences and potentially limits our understanding of cognitive phenomena in living systems, we should be careful to use such artificial systems to constrain cognitive theorizing, and remain critical about including AI cases in the target domain.

One possible response is to exclude AI systems. A more open position is to put judgment on hold for the time being, neither accepting nor excluding them. This openended position also fits the general approach taken here not to exclude potential cases a priori and acknowledges current practices. Three considerations further support the latter option. First, the general relevance of AI systems and computational models for and within the cognitive sciences is beyond doubt. They provide a key tool for investigation as well as a scientific target in their own right. In addition, taking the biogenic (human-derived) background of artifacts into account, AI systems can play their own role in human niche construction and the ongoing cyborgization of humans (Clark 2008). Finally, a more pragmatic argument is that the current proposal does not require that it is first generally accepted within the cognitive sciences. Its primary focus is to open up and extend the cognitive domain rather than to restrict it or challenge existing work. Its driving force is the conviction that we will learn many new things about cognitive phenomena by studying their occurrence in a wide range of living systems. The impact of such work on AI cases, as well as our understanding of highlevel human cognition can be evaluated at some later stage.

\section{Cobolism: the tools of life}

When living systems constitute the material foundation of cognitive phenomena, which aspects of living systems would be specifically relevant? In addition, how would this foundation impact on our understanding of cognition? To address the first question, I introduce a new concept, cobolism, to refer to the general means by which living systems manipulate and change the world. In the next section, a sketch is given how 
this proposal can change and diversify our understanding of cognitive phenomena. The cobolism proposal builds on biological work on the characteristics of life (e.g. Gánti 1975; Letelier, Cárdenas and Cornish-Bowden 2011; Noble 2013; Lane 2016; Bechtel 2011), is inspired by theoretical work on the mind-life relation within the autopoietic traditions, including autonomy, organizational and enactive approaches (e.g. Maturana and Varela 1980; Thompson 2007; Moreno and Mossio 2015; Di Paolo et al. 2017), and is part of more general, biologically-oriented approaches to cognition (GodfreySmith 2002, 2016a, b; Lyon 2006, 2015; Van Duijn et al. 2006; Keijzer 2017; Allen 2017)

Within the autopoietic traditions, much work has been done to conceptualize mind, cognition and related notions in ways that connect them to life in a fundamental conceptual way. This project is often considered problematical as there does not seem to be a fundamental conceptual connection between mind and cognition, and life (e.g. Thompson 2007; Hutto and Myin 2013; De Jesus 2016; Degenaar and O'Regan 2017). The present proposal sidesteps these conceptual problems by explicitly shifting the direction of fit between cognition and living systems. Instead of a conceptual connection, an empirical posit is made: Living systems are the natural organizations exhibiting cognitive phenomena and therefore we can expect to come to understand cognitive phenomena better by studying living systems.

Following the shared empirical and theoretical insights from the mentioned domains, living systems are conceptualized as intrinsically active, cyclically organized, self-organizing and self-producing organizations. The goal here is to articulate where cognition (as signaled by the toolkit criteria) comes in within this more general view. To repeat, the aim is not to define or describe cognition but to specify the domain that sets material constraints on cognition.

The proposal starts with metabolism, which can be taken as the core of living systems. At a fundamental level, living systems consist of complex, cyclically organized sets of biochemical processes. These biochemical processes, together with their products, such as complex molecules, membranes, tissues and extracellular structures (like extracellular matrix (ECM), bone and shells), constitute each individual living organism. Metabolism is a central, generally used concept that refers to this integrated and cyclically organized network of biochemical reactions that together fulfil the biochemical requirements of any living organization.

This cyclic network of metabolic reactions needs active maintenance, as its constituting processes only take place in circumstances that that are far from a thermodynamic equilibrium. The network's operation will depend on the availability of energy that drives its chemical reactions, on the in- and outflow of useful substances and waste products, on a chemical context that does not interfere with the metabolic processes, and on physical conditions that remain within certain boundaries as not to disturb the physical integrity of the metabolic processes. Therefore, the metabolic organization's ongoing-ness depends on contextual features that provide and regulate the conditions that enable these same metabolic processes. In various theories about the origins of life, such non-metabolic conditions, like containing structures and a flow of metabolically essential chemicals, are envisioned as being initially provided by preexisting physical structures like deep-sea hydrothermal vents (Lane 2016). However, the metabolic organization of current living systems is not similarly dependent on such 
Fig. 3 A living system, constituted and maintained by cyclic metabolic processes, which in turn depends on and maintains a cobolism: a set of interactive structures, processes and events that maintains the conditions for the ongoing existence of the metabolic organization

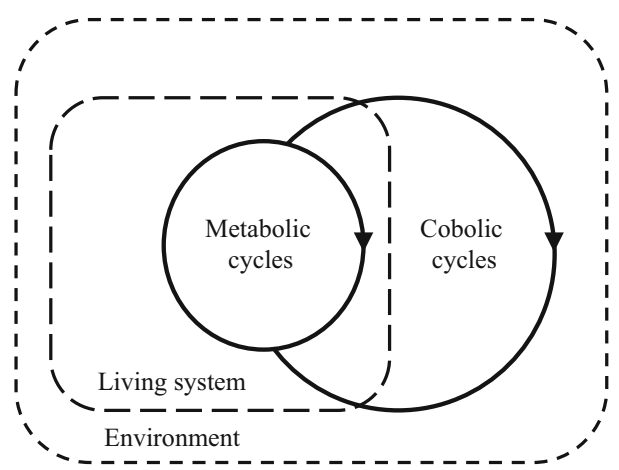

preexisting external settings. Instead, they organize their own structures, processes and happenings that maintain the conditions for their metabolic organization.

When we talk about living systems, we refer thus not only to a network of metabolic processes but also to a supportive set of tools that manipulate and organize the part of the world that contains the metabolic core and, to some extent, its neighborhood, for example by constructing a cell wall that restricts the movement of chemicals. While these tools can be very diverse, being constituted by a broad variety of structures, processes and events, they are brought together and held together in a cyclic organization that as a whole systematically maintains the internal and external conditions that enable metabolic processes to continue. I will use the phrase cobolism to refer to and conceptually bind together this additional cyclic organization (Fig. 3).

In the past, various other concepts have been introduced with somewhat similar meanings, such as structural coupling (Maturana and Varela 1980) and the interactive domain (Moreno and Mossio 2015) among others. Cobolism is related to such proposals but without a commitment to a specialized theoretical perspective, like autopoiesis or theoretical work on autonomy. Like metabolism, cobolism is a more general term intended for use in an empirical context. Cobolism is also an expandable concept that fits the various cobolic organizations found in nature. The strongest theoretical commitment is it having a cyclic organization, which ties otherwise unconnected structures, processes and events together into units that perform specific cobolic functions. Such cobolic cycles generate a living system's interactions with the world, inside and outside.

Thus, the word 'cobolism' is intended to be complementary as well as analogous to metabolism. It is complementary as a co-(meta)bolism that connects and anchors the metabolic basis of a living system within its wider environment. It is analogous as it also refers to a systematic set of cyclically organized features that are characteristic for particular lifeforms and the systematic ways they deal with their environments. Such cobolic features can involve structural parts of the living organization, e.g. a nervous system or leaf structure; processes such as sensorimotor relations, substance secretion for signaling or directed growth; and also recurring events that are brought about by and significant for maintaining the living system, such as prey capture. In this way, cobolism can play a conceptual role that has similarities to metabolism, both being general ways to systematically group a broad diversity of self-maintaining processes 
in a way that stresses their wider relevance for maintaining the living system. The conceptual similarity between metabolism and cobolism also extends to the idea that both notions refer to a systematic and cohering repertoire of processes that together constitute respectively the chemical and the interactive tools on which the organism depends for its existence.

Cobolism provides a proper target domain for developing an updated and adequate account of what we take cognition to be. While cobolism provides a much larger target domain compared to dominant views, it should be judged for its usefulness for fitting existing cases and suggesting new research on phenomena that have cognitive characteristics irrespective whether we are currently willing to call these phenomena cognitive or not.

\section{Cobolic diversity: no mere agents any more}

The proposal states that a cobolic organization provides a material basis, and thus a set of phenomena, to which the concept of cognition should be adapted. To illustrate its potential to change our thinking about cognition, I will here compare how cobolic descriptions diverge from the ascription of concepts like 'agent' and 'agency'.

In our intuitive conceptual framework, we have central concepts like mind, cognition and agents, where agents are organizations that perceive and act in some form, while they may exhibit cognition or even a mind, if they are smart enough. The concept of 'agent' is applied very widely_ranging from organisms, robots, software to groups - and its use is very little constrained by any substantial and specific form of organization (Wooldridge and Jennings 1995). Its relevance here is its role as a potential target for ascribing mind and cognition. Independent of a cobolic organization, the living system depicted in Fig. 3 can be interpreted as an agent using a sensorimotor loop to interact with its environment. Under an agentive interpretation, Fig. 3 applies as easily to an animal as to a bacterium. Both function as agents dealing with an environment while only the specific means for doing so are different. In contrast, while Fig. 3 can be taken to provide an idealized sketch of a single bacterium's cobolism, it does not reflect the animal condition, which is a much more complex multicellular organization. $^{2}$

Cobolism is defined as being centered on a metabolic organization for which it constitutes the self-build physical context and the interactive processes that help maintain it against degradation. Cells are here key building blocks. Aggregations of cells may be combined into integrated multicellular organizations like animals (Arnellos et al. 2014), and these can have new and different cognitive features both in their component systems and as a whole. Going from a unicellular to a multicellular configuration is a major change when it comes to a cobolic organization. Keeping such multicellular organizations coordinated and unified is a major task that comes with its own challenges and need for solutions (Keijzer et al. 2013). From this perspective, the work on inbodied cognition discussed in Sect. 2 is only the tip of a large cognitive iceberg awaiting further investigation.

\footnotetext{
2 In the following, I will ignore the additional issues that derive from the differences between a prokaryotic (bacteria) and a eukaryotic (animal) cell organization.
} 


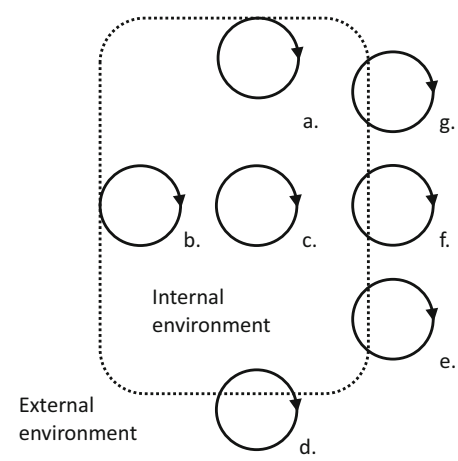

Fig. 4 An impression of the animal cobolic organization: a Combined cobolic cycles of the individual cells constituting the animal; $\mathbf{b}$ Developmental organization involved in generating and maintaining the multicellular physiological organization; c Physiological organization underlying combined cobolism; d Chemical and energetic exchanges between the multicellular organization and the multicellular environment (e.g. lungs and gut); e First-level sensorimotor cobolism; f Second-level sensorimotor cobolism; g Third-level sensorimotor cobolism

Figure 4 provides an impression of the cobolic organization that animals bring to the table to act as unitary macroscopic agents. At a basic level there is the combined individual cobolism of the constituting cells; then a developmental organization is active to build and maintain the aggregate; in addition, a physiological organization ensures general housekeeping and balances the energy requirements for various activities; clearly, centralized chemical and energetic exchanges with the extra-animal environment need to be taken care off as well; and finally sensorimotor activity itself can be subdivided in different levels, each dealing with different aspects of interacting with the environment at a whole-body level.

When looking at its cobolism, an animal is not a basic cognitive organization. Its macroscopic perceptual and behavioral characteristics derive from and contribute to a complex underlying cobolic organization build around a basic core of metabolic biochemical processes. This cobolic organization enables an agglomeration of cells to act as a macroscopic single unit. The presence of an extensive animal-style cobolic organization is also a precondition for becoming capable of perceiving and acting on the macroscopic world with its surfaces and objects that we, as humans, are most familiar with, being ourselves such extensive multicellular organizations (Keijzer 2015). In this view, the macroscopic world of surfaces and objects is not a starting point, but rather a target for cognitive explanations.

Cobolism not only expands into multicellular forms-where plants and fungi will have their own multicellular organizations-but also allows social forms, symbiosis and extensive forms of niche construction. There is also no reason to exclude the special characteristics of human social organization, language, reasoning and technology. Such human specialties do fit in this way of thinking, which is essentially open ended and descriptive. How the cognitive sciences might want to carve up and organize such a huge and diverse domain is an issue to be addressed by these various sciences themselves, and a matter of later concern. 
By grounding cognition in the cobolism of living organizations, the notion of cognition itself can be developed in ways that go beyond the classic and intuitive agent/mind-environment format. Atypical cognitive cases such as plants and blood vessel cells can be accommodated by describing the cobolic organization they constitute or are a part of. Cobolism provides a framework that accepts the organization of the interactive structures that living systems possess without strong theoretical commitments or judgments concerning these structures. In this way it is open to expand its set of descriptions to incorporate the broad variety that can be encountered in the world.

\section{Reprise: "But is it really cognition?"}

Since the cognitive revolution within and around psychology, 'cognition' has become a standard word to refer to the processes that make us-humans-intelligent. In this paper, I tried to reorient the way in which we think about those processes by cutting them free from the mental conceptual domain and grounding them in an empirical domain that I described above as a cobolism: the integrated set of internal and external structures, processes and events that are present in and used by living systems to maintain the fundamental metabolic processes that sit at their core.

Two central ideas drove this proposal. First, the discovery of a broad variety of atypical forms of cognitive explananda, as signaled by the presence of cognitive toolkit criteria such as sensing, valence, behavior, memory, learning, anticipation, decision making and communication. These atypical cases come from all areas of life and set up new targets for an up-to-date account of cognition. Second, the notion of cognition is already in flux and changing to accommodate new findings and insights. Such changes are normal for scientific terms, which over the years can diverge from initial interpretations. Tying cognition to the systems that naturally exhibit cognitive processes and adapt the notion of cognition itself to fit these empirical cases is a standard way of doing science.

Does this proposal diverge too far from conventional views that limit the use of cognition to human style reasoning? There are two issues here: How do we apply the word 'cognition', and to what extent do we need to separate human-level cognition from this wider range of cases? The first is ultimately a minor issue. The wider usage of the word 'cognition' is important to signal the cognitive relevance of living systems in general, but if it is deemed necessary, other words might be used and the proposal would still stand.

The perceived need to separate the human case from other (potential) cases of cognitive phenomena is a key issue though. This paper explicitly opposes such a fundamental separation. It highlights the empirical possibility that human reasoning is ultimately a specific and complex form of processes that are widely present in living systems. This possibility must not be excluded without further study of these cases. Of course, there are major differences too, but to assess the extent, importance and nature of these differences, we should weigh them against the similarities. Thus, irrespective of what we currently or intuitively think of the position of human reasoning, it will be important to pay attention to cognition widely conceived. Studying a wide range 
of cognitive phenomena is the key to provide better ideas and more relevant findings concerning the status of human reasoning too. So, even those who are convinced that the study of human cognition is fundamentally different from non-human cases, to articulate and establish such a strong and far-reaching claim will require work on 'real' as well as 'unreal' cognition, giving rise to what we can call the cognitive life sciences.

Acknowledgements I want to thank the reviewers for their comments and help to make this a better paper. I also want to thank Jan Degenaar, Boris Demarest, Joris Van Poucke, Gertrudis Van de Vijver, Erik Myin, Hanno Sauer, Jasper van den Herik, Fred Muller, Marc van Duijn, Corijn van Mazijk and Farid Zahnoun for the feedback provided by their commentaries on a target paper in Dutch 'De intuitties voorbij: Een biologische interpretatie van cognitie' (ANTW (2016) 108, no. 2) that provided the starting point for this paper.

Open Access This article is licensed under a Creative Commons Attribution 4.0 International License, which permits use, sharing, adaptation, distribution and reproduction in any medium or format, as long as you give appropriate credit to the original author(s) and the source, provide a link to the Creative Commons licence, and indicate if changes were made. The images or other third party material in this article are included in the article's Creative Commons licence, unless indicated otherwise in a credit line to the material. If material is not included in the article's Creative Commons licence and your intended use is not permitted by statutory regulation or exceeds the permitted use, you will need to obtain permission directly from the copyright holder. To view a copy of this licence, visit http://creativecommons.org/licenses/by/4.0/.

\section{References}

Adams, F. (2018). Cognition wars. Studies in History and Philosophy of Science, 68, $20-30$.

Adams, F., \& Aizawa, K. (2001). The bounds of cognition. Philosophical Psychology, 14, 43-64.

Adams, F., \& Garrison, R. (2013). The mark of the cognitive. Minds and Machines, 23(3), 339-352.

Akagi, M. (2018). Rethinking the problem of cognition. Synthese, 195(8), 3547-3570.

Allen, C. (2017). On (not) defining cognition. Synthese, 194(11), 4233-4249.

Anderson, M. L. (2015). Mining the brain for a new taxonomy of the mind. Philosophy Compass, 10(1), 68-77.

Arnellos, A., Moreno, A., \& Ruiz-Mirazo, K. (2014). Organizational requirements for multicellular autonomy: Insights from a comparative case study. Biology and Philosophy, 29(6), 851-884.

Baluška, F., \& Levin, M. (2016). On having no head: Cognition throughout biological systems. Frontiers in Psychology, 7, 902.

Baluška, F., Mancuso, S., Volkmann, D., \& Barlow, P. (2009). The 'root-brain' hypothesis of Charles and Francis Darwin: Revival after more than 125 years. Plant signaling \& behavior, 4(12), 1121-1127.

Barrett, L. (2015). A better kind of continuity. Southern Journal of Philosophy, 53, $28-49$.

Bayne, T., Brainard, D., Byrne, R. W., Chittka, L., Clayton, N., Heyes, C., et al. (2019). What is cognition? Current Biology, 29, R603-R622.

Bechtel, W. (2011). Mechanism and biological explanation. Philosophy of Science, 78(4), 533-557.

Ben-Jacob, E., Becker, I., Shapira, Y., \& Levine, H. (2004). Bacterial linguistic communication and social intelligence. Trends in Microbiology, 12(8), 366-372.

Bentley, K., \& Chakravartula, S. (2017). The temporal basis of angiogenesis. Philosophical Transactions $B, 372(1720), 20150522$.

Bentley, K., Philippides, A., \& Regan, E. R. (2014). Do endothelial cells dream of eclectic shape? Developmental Cell, 29(2), 146-158.

Boden, M. (2006). Mind as machine: A history of cognitive science (two volumes). Oxford: OUP.

Brembs, B. (2009). The importance of being active. Journal of Neurogenetics, 23(1-2), 120-126.

Buckner, C. (2015). A property cluster theory of cognition. Philosophical Psychology, 28(3), 307-336.

Burge, T. (2014). Perception: Where mind begins. Philosophy, 89(03), 385-403.

Carruthers, P. (2004). On being simple minded. American Philosophical Quarterly, 41(3), 205-220.

Chittka, L. (2017). Bee cognition. Current Biology, 27(19), R1049-R1053. 
Clark, A. (2008). Supersizing the mind. Oxford: OUP.

Clark, A. (2011). Finding the mind. Philosophical Studies, 152(3), 447-461.

Clark, A., \& Chalmers, D. (1998). The extended mind. Analysis, 58, 7-19.

Davidson, D. (1999). The emergence of thought. Erkenntnis, 51, 7-17.

De Jesus, P. (2016). Autopoietic enactivism, phenomenology and the deep continuity between life and mind. Phenomenology and the Cognitive Sciences, 15(2), 265-289.

Degenaar, J., \& O’Regan, J. K. (2017). Sensorimotor theory and enactivism. Topoi, 36(3), 393-407.

Di Paolo, E. A., Buhrmann, T., \& Barandiaran, X. (2017). Sensorimotor life: An enactive proposal. Oxford: OUP.

Etxebeste, O., \& Espeso, E. A. (2016). Neurons show the path: Tip-to-nucleus communication in filamentous fungal development and pathogenesis. FEMS Microbiology Reviews, 40(5), 610-624.

Figdor, C. (2013). What is the "cognitive" in cognitive neuroscience? Neuroethics, 6(1), 105-114.

Figdor, C. (2018). Pieces of mind: The proper domain of psychological predicates. Oxford: OUP.

Gánti, T. (1975). Organization of chemical reactions into dividing and metabolizing units: The chemotons. BioSystems, 7(1), 15-21.

Gao, T., Newman, G. E., \& Scholl, B. J. (2009). The psychophysics of chasing: A case study in the perception of animacy. Cognitive Psychology, 59(2), 154-179.

Gardner, H. (1987). The mind's new science: A history of the cognitive revolution. New York: Basic books.

Godfrey-Smith, P. (2002). Environmental complexity and the evolution of cognition. In R. J. Sternberg \& J. C. Kaufman (Eds.), The evolution of intelligence (pp. 233-249). London: Psychology Press.

Godfrey-Smith, P. (2016a). Mind, matter, and metabolism. Journal of Philosophy, 113(10), 481-506.

Godfrey-Smith, P. (2016b). Individuality, subjectivity, and minimal cognition. Biology and Philosophy, 31(6), 775-796.

Green, C. D. (1996). Where did the word "cognitive" come from anyway? Canadian Psychology, 37, 31-39.

Hamlyn, D. W. (1994). Perception. In S. Guttenplan (Ed.), A companion to the philosophy of mind (pp. 459-463). Oxford: Blackwell.

Hurley, S. L. (1998). Consciousness in action. Harvard: Harvard University Press.

Hurley, S. L., \& Nudds, M. (Eds.). (2006). Rational animals?. Oxford: OUP.

Hutto, D. D., \& Myin, E. (2013). Radicalizing enactivism: Basic minds without content. Cambridge: MIT Press.

Jékely, G., Keijzer, F. A., \& Godfrey-Smith, P. (2015). An option space for early neural evolution. Philosophical Transactions of the Royal Society B, 370, 201550181.

Kaplan, D. M. (2012). How to demarcate the boundaries of cognition. Biology and Philosophy, 27(4), $545-570$.

Keijzer, F. A. (2001). Representation and behavior. Cambridge: MIT Press.

Keijzer, F. A. (2015). Moving and sensing without input and output: Early nervous systems and the origins of the animal sensorimotor organization. Biology and Philosophy, 30(3), 311-331.

Keijzer, F. A. (2017). Evolutionary convergence and biologically embodied cognition. Interface Focus, 7(3), 20160123.

Keijzer, F. A., Van Duijn, M., \& Lyon, P. (2013). What nervous systems do: Early evolution, input-output, and the Skin Brain Thesis. Adaptive Behavior, 21(2), 67-84.

Lane, N. (2016). The vital question: Why is life the way it is?. London: Profile Books.

Letelier, J. C., Cárdenas, M. L., \& Cornish-Bowden, A. (2011). From L'Homme Machine to metabolic closure: Steps towards understanding life. Journal of Theoretical Biology, 286, 100-113.

Levin, M. (2013). Reprogramming cells and tissue patterning via bioelectrical pathways: Molecular mechanisms and biomedical opportunities. Wiley Interdisciplinary Reviews: Systems Biology and Medicine, 5(6), 657-676.

Levin, M., \& Martyniuk, C. J. (2018). The bioelectric code: An ancient computational medium for dynamic control of growth and form. BioSystems, 164, 76-93.

Lyon, P. (2006). The biogenic approach to cognition. Cognitive Processing, 7(1), 11-29.

Lyon, P. (2015). The cognitive cell: Bacterial behavior reconsidered. Frontiers in Microbiology, 6, 264.

Lyon, P. (2019). Of what is "minimal cognition" the half-baked version? Adaptive Behavior, 1059712319871360.

Macnab, R. M., \& Koshland, D. E. (1972). The gradient-sensing mechanism in bacterial chemotaxis. Proceedings of the National Academy of Sciences, 69(9), 2509-2512.

Mancuso, S., \& Viola, A. (2015). Brilliant green: The surprising history and science of plant intelligence. Washington, DC: Island Press. 
Manicka, S., \& Levin, M. (2019). The Cognitive Lens: A primer on conceptual tools for analysing information processing in developmental and regenerative morphogenesis. Philosophical Transactions of the Royal Society B, 374(1774), 20180369.

Maturana, H. R., \& Varela, F. J. (1980). Autopoiesis and cognition: The realization of the living. Dordrecht: Reidel.

Moreno, A., \& Mossio, M. (2015). Biological autonomy: A philosophical and theoretical enquiry. Berlin: Springer.

Newen, A. (2017). What are cognitive processes? An example-based approach. Synthese, 194(11), 4251-4268.

Noble, D. (2013). Physiology is rocking the foundations of evolutionary biology. Experimental Physiology, 98(8), 1235-1243.

O'Regan, J. K., \& Noë, A. (2001). A sensorimotor account of vision and visual consciousness. Behavioral and Brain Sciences, 24(05), 939-973.

Parniske, M. (2008). Arbuscular mycorrhiza: The mother of plant root endosymbioses. Nature Reviews Microbiology, 6(10), 763-775.

Pfeifer, R., \& Bongard, J. (2007). How the body shapes the way we think: A new view of intelligence. Cambridge: MIT Press.

Poldrack, R. A. (2006). Can cognitive processes be inferred from neuroimaging data? Trends in Cognitive Sciences, 10, 59-63.

Poldrack, R. A. (2010). Mapping mental function to brain structure: How can cognitive neuroimaging succeed? Perspectives on Psychological Science, 5, 753-761.

Ramsey, W. (2017). Must cognition be representational? Synthese, 194(11), 4197-4214.

Reid, C. R., \& Latty, T. (2016). Collective behaviour and swarm intelligence in slime moulds. FEMS Microbiology Reviews, 40(6), 798-806.

Rosslenbroich, B. (2014). On the origin of autonomy: A new look at the major transitions in evolution. Berlin: Springer.

Rowlands, M. (2010). The new science of the mind: From extended mind to embodied phenomenology. Cambridge: MIT Press.

Rumelhart, D. E., McClelland, J. L., \& The PDP research group. (1986). Parallel distributed processing: Explorations in the microstructure of cognition. Part 1, Foundations. Cambridge: MIT Press.

Scholl, B. J., \& Tremoulet, P. D. (2000). Perceptual causality and animacy. Trends in cognitive sciences, 4(8), 299-309.

Searle, J. R. (1983). Intentionality: An essay in the philosophy of mind. Cambridge: Cambridge University Press.

Serrano, J. I., del Castillo, M. D., \& Carretero, M. (2014). Cognitive? Science? Foundations of Science, 19(2), 115-131.

Stewart, J. (1996). Cognition = life: Implications for higher-level cognition. Behavioural Processes, 35(1), 311-326.

Stoecker, R. (2009). Why animals can't act. Inquiry, 52(3), 255-271.

Thompson, E. (2007). Mind in life: Biology, phenomenology, and the sciences of mind. Harvard: Harvard University Press.

Trewavas, A. (2005). Green plants as intelligent organisms. Trends in Plant Science, 10(9), 413-419.

Turing, A. M. (1950). Computing machinery and intelligence. Mind, 59(236), 433-460.

Van Duijn, M., Keijzer, F. A., \& Franken, D. (2006). Principles of minimal cognition: Casting cognition as sensorimotor coordination. Adaptive Behavior, 14(2), 157-170.

Van Gelder, T. (1995). What might cognition be, if not computation? Journal of Philosophy, 92(7), 345-381.

Van Loon, L. C. (2016). The intelligent behavior of plants. Trends in Plant Science, 21(4), 286-294.

Varela, F., Thompson, E., \& Rosch, E. (1991). The embodied mind. Cambridge: MIT Press.

Walter, S., \& Kästner, L. (2012). The where and what of cognition: The untenability of cognitive agnosticism and the limits of the Motley Crew Argument. Cognitive Systems Research, 13(1), 12-23.

Westerhoff, H. V., Brooks, A. N., Simeonidis, E., García-Contreras, R., He, F., Boogerd, F. C., et al. (2014). Macromolecular networks and intelligence in microorganisms. Frontiers in Microbiology, 5, 379.

Wooldridge, M., \& Jennings, N. R. (1995). Intelligent agents: Theory and practice. Knowledge Engineering Review, 10(2), 115-152.

Publisher's Note Springer Nature remains neutral with regard to jurisdictional claims in published maps and institutional affiliations. 